\title{
Mapping of Acoustic Noise and Microwave Radiation
}

\author{
Volodymyr V. Kudriashov ${ }^{1}$, Artem Yu. Garbar ${ }^{2}$, Sergii K. Lukin ${ }^{3}$, \\ Volodymyr P. Palamarchuk ${ }^{4}$, Konstantin A. Lukin ${ }^{4}$ \\ ${ }^{1}$ Institute of Information and Communication Technologies, BAS, 1113 Sofia, Bulgaria \\ 2 National Technical University "Kharkiv Polytechnic Institute", 21 Frunze str., 61002 Kharkiv, \\ Ukraine \\ ${ }^{3}$ Parthenope University of Naples, 38 Amm. F. Acton str., 80133 Naples, Italy \\ ${ }^{4}$ O.Ya. Usikov Institute for Radiophysics and Electronics, National Academy of Sciences of Ukraine, \\ 12 Ak. Proskura str., 61085 Kharkiv, Ukraine \\ Emails:KudriashovVladimir@Gmail.com_Sergiy.Lukin@UniParthenope.itrPalmarchuk@Mail.ru \\ Lukin.Konstantin@Gmail.com
}

\begin{abstract}
Passive sensor systems are attractive for monitoring due to the absence of probing emissions. Two passive systems: an acoustic camera, manufactured by Brüel \& Kjor (Sound and Vibration Measurement A/S) and a microwave radiometer are considered in the paper. The performance of these systems is carefully analyzed. The obtained results show the limitations of the acoustic camera in acoustic noise source localization caused by specific side lobes of a pattern of its microphone array. A suggestion how to avoid them is given. Application of microwave bistatic radiometer provides information about thermal electromagnetic radiation of objects in $3 D$ spatial coordinates. The generated radiometric maps contain amplitude and phase information, which is suitable for estimation of displacements/vibration. The received results are applicable in nondestructive testing.
\end{abstract}

Keywords: Acoustic camera, beamforming, microwave radiometer, bistatic radiometer, experimental results.

\section{Introduction}

The first acoustic camera system was described in the early 80th when Billingsley [1] proposed an acoustic telescope. Two years later, Billingsley and Kinns [2] have developed a full-scale system for real-time sound source location of full-size jet engines. This first system consisted of an array of microphones connected to a small digital computer, via a sequence of preamplifiers, analog filters and analog to digital converters and multiplexer. The 
computer processed in real time microphone signals and visualized source distributions on a color display with respect to acoustic source position and frequency. The system was designed to be mobile and easily transported. Since that time, the architecture of acoustic camera system remains approximately the same. The enhancements mostly consider the performance of sensors (microphones), ADC frequency, antenna design, and processing software.

Techniques for generation of acoustic maps restrict the signal frequency bandwidth and limit the resolution of the maps. A vibration of objects may be estimated with non-acoustic techniques. Radiometric monitoring allows obtaining information about natural thermal electromagnetic radiation of objects at a physical temperature above $0 \mathrm{~K}$ [3]. The spatial resolution of the radiometer is usually based on parameters of its real or synthesized antenna beam [4]. However, the spatial diversity of the bistatic radiometer antennas enables to estimate additionally Time Difference Of Arrival (TDOA) of objects thermal emissions [3]. Objects' vibration detector may be based on Doppler filter bank on the output of the bistatic radiometer. Thus, the vibration frequency and the sensor beam width become independent. Moreover, all vibration frequencies may be obtained simultaneously, without repetitive acoustic estimates for different frequency bands. The bistatic radiometer's integration time up to one second corresponds to narrow width of Doppler filters. Obtained information on the vibration may be useful for acoustic noise source localization.

The next chapter consists of some preliminary information about algorithms for signal processing in acoustic camera systems. Then follows a description of tested equipment purchased from Brüel \& Kjaer. The received results are provided in the next chapter. A description of the bistatic radiometer is given below. Lastly, some conclusions are summarized.

\section{Visualizing of sounds using acoustic camera}

The acoustic camera uses two different types of sensors - camera and microphones. Acoustic camera fuses image received by the camera with the data by microphones in a complex image. This image consists of camera image as a background and contour lines describing a sound field as a foreground. To join these very different data, a complicated processing has to be applied over raw signal data received by a microphone array. Information for source location and source spectral characteristics has to be determined.

Two different techniques are applied to measure sound field parameters. The first one - Near-field Acoustical Holography (NAH), proposed in 1980 by M a y a rd, Willi a m s and Le e [5], follows the principle of optical holography, invented by Dennis Gabor in 1948. The 2D recording of sound waves (phase and amplitude), called hologram, is used to reconstruct the 3D sound field. NAH may be used for generation of calibrated maps of sound intensity, sound pressure and particle velocity. The main requirements to the NAH acoustic array are to be placed close to the observed source and to be larger than the target of interest. At high frequencies and for the case of large objects the number of measurement points 
becomes enormous. This can create a number of problems regarding the design of such multi-channel system or regarding validity of assumption concerning stationarity of prolonging measurement process when scanning of the object by a smaller antenna is used.

The second technique is realized through BeamForming (BF). BF is a spatial filtering of signals from/to sensor array in order to carry out directional signal reception/transmission. BF is closely related with a development of phased array transmission in 1905 by Karl Ferdinand Braun, who described in his Nobel lecture a 3-element antenna with directed emission [6]. The BF received wider practical use in radar systems during World War II. In acoustics, the BF is used to localize sound sources, on medium to long measurement distances. The angular resolution depends mainly from antenna size and wave frequency. At low frequency, the antenna size has to be bigger to assure good angular resolution. The sound map is estimated focusing the antenna onto the pixels in a plane, parallel to the antenna plane on a given distance. For each point, a weighted sum of individual microphone delayed signals is received (so-called Delay and Sum Beamforming). The time delay for each microphone depends on the 3D positions of the estimated point and the microphone under consideration. The time delay is calculated in correspondence with the distance from estimated point to the microphone.

The quality of BF depends on resolution and maximum sidelobe levels. The resolution is determined by the smallest angular separation of two point sources allowing to be seen as two points. The maximum sidelobe levels will be described by antenna direction pattern. The direction pattern characterizes with one or more main beam/beams (or lobe/lobes) with maximum signal amplification and many others side beams with lower signal amplification. The side beams produce false peaks/sources. The maximum sidelobe levels, measured relatively to the main lobe define the probability of appearing of ghost sources.

The BF technique characterizes with good quality maps for objects on middle and far distance. In spite of NAH its quality enhances for higher frequencies, but diminishes for lower ones, when the NAH works well. The BF allows implementation of irregular antenna arrays that generally outperform regular arrays reducing an aliasing problem.

\section{Acoustic camera equipment}

The Brüel \& Kjaer acoustic camera includes a non-uniform slice wheel microphone array type WA-1558-W-021 [7, 8]. The diameter of the array is about $0.33 \mathrm{~cm}$. The number of microphones in the array is 18 .

The microphone has built-in CCLD preamplifier with transducer electronic datasheet (TEDS-IEEE1451.4 V.1.0). The microphone dimensions are $34 \mathrm{~mm}$ long, $7 \mathrm{~mm}$ diameter. The microphone sensitivity is $11.2 \mathrm{mV} / \mathrm{Pa}$. The operating temperature range is from $-10^{\circ} \mathrm{C}$ up to $+55^{\circ} \mathrm{C}$. The microphone dynamic range is from $28 \mathrm{~dB}$ up to $140 \mathrm{~dB}$.

The 6-channel input module delivers Dyn-X technology, which expands its dynamic range. The exact dynamic range value depends on the signal quantization 
and bandwidth. The 12-channel input module has no Dyn-X technology. Both input modules support TEDS transducers; they deliver REq-X technology which flattens the result by "mirroring" the frequency responses. These input modules are mounted in 5-slot Mainframe LAN-XI type 3660-C-000 with battery module type 2831.

Amplitude calibration can be provided in order to make precise power estimation. The pistonphone calibrator type 4228 satisfies IEC 942 (1988) Class 1L or Class 0L (with external barometer) and ANSI S1.40-1984. Adaptor DP-0775 is suitable to perform sequential calibration of all these microphones. It can be performed a fast calibration of 6-microphones using adaptor WA-0728-W-003. The calibration frequency is $251.2 \mathrm{~Hz}$. The calibrator is battery operated. The calibrator delivers high frequency and level stability. It can be used in the field over a wide range of temperature, humidity and pressure while still maintaining high accuracy.

Data transfer cables limit the spatial separation of equipment blocks. The acoustic camera consists of three main blocks: a microphone array with an optic camera, input modules in a frame and a laptop with software. Microphone array data is transferred to input modules with the cable harness WL1297-W-004 2013W21 which length is about $4.5 \mathrm{~m}$. The acquired data is transferred to the laptop with LAN cable AO1450-D-020 2013W13 which length is about $2 \mathrm{~m}$. The optic camera data is transferred to the laptop with USB cable which length is about $6.3 \mathrm{~m}$. Optic camera resolution is $640 \times 480$ points. The main features of the laptop type 7201-E-GB2 (Dell Latitude E6430) are listed below: E6430 CPU, 6 GB RAM, 1 TB HDD, Wi-Fi, Ethernet 1 Gb, DVD-RW.

The both microphone array and optic camera are mounted on tripod Manfrotto 058B and 3D tripod head Manfrotto 229. The load capacity of both the tripod and the head is $12 \mathrm{~kg}$ (safety payload).

The acoustic camera is supplied with full documentation (instruction manual, specifications) to hardware and software modules. Measurement data can be collected with Pulse LabShop (Customized Solution Version 17.1.2). Acoustic maps can be formed with Array Acoustics Post-processing (Version 17.1.2.308). Other existing software and drivers are not listed in this work.

The frequency characteristics given by the acoustic camera manufacturer Brüel \& Kjær (Sound and Vibration Measurement $\mathrm{A} / \mathrm{S}$ ) are given in Table 1. The quantization rate is $65536 \mathrm{~Hz}$. The acoustic camera upper frequency is $25.6 \mathrm{kHz}$. The sampling ratio is 2.56. Synchronization of samples of input modules is provided by using IEEE 1588 Precision Time Protocol [9].

Table 1. Frequency characteristics for modules of the acoustic camera

\begin{tabular}{|c|c|c|l|}
\hline \multirow{2}{*}{ Name } & Type & $\begin{array}{c}\text { Frequency range, } \\
\mathrm{kHz}\end{array}$ & \multicolumn{1}{c|}{ Features } \\
\hline Microphones & 4958 & $0.01-20$ & \multicolumn{1}{c|}{-} \\
\hline \multirow{2}{*}{ Input Modules } & $3053-\mathrm{B}-120$ & $0-25.6$ & $\begin{array}{l}\text { 1. Sampling rate: } 65.5 \mathrm{k} \text { samples } \\
\text { per s } \\
\text { 2. Number of input channels 12 }\end{array}$ \\
\cline { 2 - 4 } & $3050-\mathrm{B}-060$ & $\begin{array}{l}\text { 1. Sampling rate 131 k samples } \\
\text { per s } \\
\text { 2. Number of input channels 6 }\end{array}$ \\
\hline
\end{tabular}




\section{Some results from indoor tests}

The generation of acoustic maps requires accumulation of incoming signals with respect to their delays caused by propagation paths to different transducers. The delay and sum algorithm (Delay And Sum - DAS BF) has to be performed in time domain. The exact implementation can be performed using analog phase shifters and electronic analog integrator. Thus, the integrator output defines signal amplitude in a several angular direction. Multiple input systems enable simultaneous (in parallel regime) estimation of such amplitudes by virtue of increasing complexity of apparatus. The analog electronic circuits' temperature drift and other factors limit apparatus performance. The digital systems alleviate most of these drawbacks. Digital multiple input system enables software implementation of shifts and integration of output signals received from the analog-to-digital converter. The latest decreases apparatus complexity thus improves the system parameters. The specially designed software is applied to estimate antenna performance in indoor conditions.

Experimental investigation of microphone array pattern is provided, in the presence of multipath propagation of acoustic waves. The experiment includes a point-like source of the noise waveform signal. The narrowband signal central frequencies are from 0.5 up to $20 \mathrm{kHz}$. The signal bandwidth equals to $10 \%$ of central frequency. The input signal power is chosen larger enough in comparison with acoustic camera intrinsic noise power. The integration time was not less than $250 \mathrm{~ms}$. Distance from the signal emitter to microphone array (about $0.7 \mathrm{~m}$ ) is comparable to the array dimensions. Indoor conditions include multiple sound reflectors like room floor, ceiling, walls, office furniture and equipment. Experimental investigations were provided with beam scanning discrete 1 degree along both azimuth and elevation directions. Experiments with scanning discrete enhanced to 0.1 degree delivered no qualitative results. The numbers from experimental results were rounded. It is worth to be mentioned that some of Side Lobe level values in tables below were obtained from Grating Lobes.

Side Lobes of antenna pattern are unwanted as well as its Grating Lobes (if exist). Known position of Grating Lobes enables to limit the field of view (opening angles). Applying the reduction of the field of view avoids visualization of unwanted peaks at angular positions which correspond to Grating Lobes. Meanwhile, the Side Lobes describe the level of reception of unwanted signals. The Side Lobes will produce ghosts on the acoustic map even in the limited field of view. In our experiments the field of view was widened to angular coordinates \pm 90 degree in both azimuth and elevation directions.

The newly developed software enabled to estimate antenna pattern. It uses a matrix-form implementation of the mentioned DAS BF and Improved BF algorithms [10]. The obtained results of DAS BF algorithm are given in Table 2. These results are time dependent: they had variation during measurement time affected by several phase deviation of equipment channels. 
Table 2. The antenna pattern obtained using DAS Beamforming algorithm

\begin{tabular}{|c|c|c|c|}
\hline \multirow{2}{*}{$\begin{array}{c}\text { Center frequency, } \\
\mathrm{kHz}\end{array}$} & \multicolumn{2}{|c|}{$\begin{array}{c}\text { Beamwidth, } \\
\text { degree }\end{array}$} & $\begin{array}{c}\text { Side lobe level, } \\
\mathrm{dB}\end{array}$ \\
\cline { 2 - 3 } & Azimuth & Azimuth & \\
\hline 0.5 & \multicolumn{2}{|c|}{140} & 3 \\
\hline 1 & 62 & 59 & 8 \\
\hline 3 & 18 & 20 & 8 \\
\hline 6 & 9 & 10 & 5 \\
\hline 10 & 7 & 8 & 5 \\
\hline 18 & 3 & 3 & 8 \\
\hline
\end{tabular}

The Side Lobes level shows the attenuation of received unwanted interference at an angular direction. The obtained Side Lobe level is up to $-3 \mathrm{~dB}$ (Table 2).

It is mentioned that intrinsic (thermal) noises of different receiving channels within transducers are independent. The Improved BF algorithm is based on an exclusion of main part of autocorrelation noise [10]. This algorithm implementation is in the frequency domain. The received signals spectra are used to generate a cross-spectral matrix. The specified above noise is concentrated in the region of main diagonal of such matrix. It is proposed to replace the diagonal elements by zeros, which enables to improve the noise level of the generated map via suppression of Side Lobe level of antenna pattern up to $0.6 \mathrm{~dB}$ [10]. The carried out numerical simulations confirmed this statement and the experimentally obtained antenna pattern parameters are given in Table 3. Moreover, the experimental investigation showed a Main Lobe improvement in the case of multipath wave propagation.

Grating Lobes of antenna patterns of the non-uniform microphone array were estimated as well. For center frequency $7 \mathrm{kHz}$, its azimuth position is 40 degree and its elevation position is 60 degree. For the frequencies $8,9,11,13,17,18$ and $19 \mathrm{kHz}$, their azimuth positions are 36, 31, 24, 20, 18, 17 and 16 degree. For the listed frequencies, their elevation positions are 50, 43, 34, 29, 28, 20 and 19 degree. These position values may vary slightly. It can be seen from Table 4 that temporal variation is 1 degree for frequency $13 \mathrm{kHz}$.

Table 3. The antenna pattern obtained by Improved BF algorithm
\begin{tabular}{|c|c|c|c|}
\hline \multirow{2}{*}{$\begin{array}{c}\text { Center frequency, } \\
\mathrm{kHz}\end{array}$} & \multicolumn{2}{|c|}{$\begin{array}{c}\text { Beamwidth, } \\
\text { degree }\end{array}$} & $\begin{array}{c}\text { Side lobe level, } \\
\mathrm{dB}\end{array}$ \\
\cline { 2 - 3 } & Azimuth & Elevation & \\
\hline 0.5 & 128 & 152 & 3 \\
\hline 1 & 57 & 58 & 11 \\
\hline 3 & 17 & 19 & 10 \\
\hline 6 & 8 & 9 & 7 \\
\hline 10 & 6 & 5 & 6 \\
\hline 18 & 3 & 3 & 7 \\
\hline
\end{tabular}

In the next experiment, the positions of Side Lobe peaks, closest to the Main Lobe, and with the level higher than $-10 \mathrm{~dB}$ are estimated (Table 5). Some of these peaks are results of multipath propagation of acoustic waves in indoor conditions. 
Table 4. Estimated positions of grating lobes

\begin{tabular}{|c|c|c|c|c|}
\hline \multirow{2}{*}{$\begin{array}{c}\text { Center frequency, } \\
\mathrm{kHz}\end{array}$} & \multicolumn{2}{|c|}{$\begin{array}{c}\text { DAS beamforming, } \\
\text { degree }\end{array}$} & \multicolumn{2}{c|}{$\begin{array}{c}\text { Improved beamforming, } \\
\text { degree }\end{array}$} \\
\cline { 2 - 5 } & Azimuth & Elevation & Azimuth & Elevation \\
\hline 10 & 27 & 38 & 27 & 26 \\
\hline 13 & 21 & 28 & 20 & 28 \\
\hline 15 & 19 & 24 & 20 & 25 \\
\hline 19 & 14 & 12 & 18 & 19 \\
\hline
\end{tabular}

Let us consider the generated acoustic map sample displayed on Fig. 1. The post-processing raw data was recorded with the acoustic camera. The Improved BF was applied to two point-like emission sources, placed at distance $0.75 \mathrm{~m}$ to the microphone array. The cross-range distance between these sources was established on $0.085 \mathrm{~m}$. Center frequency of these noise waveform sources was chosen $10 \mathrm{kHz}$.

Table 5. Positions of side lobes peaks, which are higher than $-10 \mathrm{~dB}$
\begin{tabular}{|c|c|c|c|c|}
\hline $\begin{array}{c}\text { Center frequency, } \\
\mathrm{kHz}\end{array}$ & \multicolumn{2}{c}{$\begin{array}{c}\text { DAS beamforming, } \\
\text { degree }\end{array}$} & \multicolumn{2}{c|}{$\begin{array}{c}\text { Improved beamforming, } \\
\text { degree }\end{array}$} \\
\cline { 2 - 5 } & Azimuth & Elevation & Azimuth & Elevation \\
\hline 1 & $>85$ & $>85$ & $>85$ & $>85$ \\
\hline 3 & $>85$ & $>85$ & $>85$ & $>85$ \\
\hline 6 & 28 & 46 & 9 & 10 \\
\hline 8 & 20 & 44 & 6 & 10 \\
\hline 10 & 18 & 35 & 27 & 26 \\
\hline 12 & 20 & 17 & 17 & 35 \\
\hline 14 & 16 & 16 & 16 & 16 \\
\hline 16 & 11 & 22 & 15 & 55 \\
\hline 18 & 13 & 24 & 14 & 67 \\
\hline 20 & 12 & 13 & 11 & 26 \\
\hline
\end{tabular}

The bandwidth was $10 \%$ of center frequency. Beam steering discrete was 1 degree. Field of view equals to above one ( \pm 90 degree). Colour bar shows the correspondence of normalized values $(\mathrm{dB})$ to the color scheme. Acoustic map pixels which level is lower than $-15 \mathrm{~dB}$ are not displayed. Red dots in the center of the map (elevation 1 degree; azimuth about 0 degree) correspond to the angular positions of these sources. The normalized acoustic map has peaks on the level $6 \mathrm{~dB}$ (Side Lobe level described in Table 3). These peaks (azimuth 20 degree and elevation 40 degree) are connected with Grating Lobes (Table 4). Side Lobes exceed $-10 \mathrm{~dB}$ level in angular range narrower than shown in Table 5 . The quality of the generated maps might be improved by application of sophisticated approaches for signal processing [11-13].

The obtained experimental results approve the correctness of system technical description. Some small differences may be observed due to indoor test scenario. The experiments show the importance of monitoring of angular directions where the high level of the unwanted signals appears. In order to minimize the number of "ghost images", the field of view of the system has to be reduced to angular coordinates given in Table 4. By the reduction, some of the interferences will not be displayed on acoustic maps as unwanted peaks but will affect these maps via Side Lobes of their own responses. 


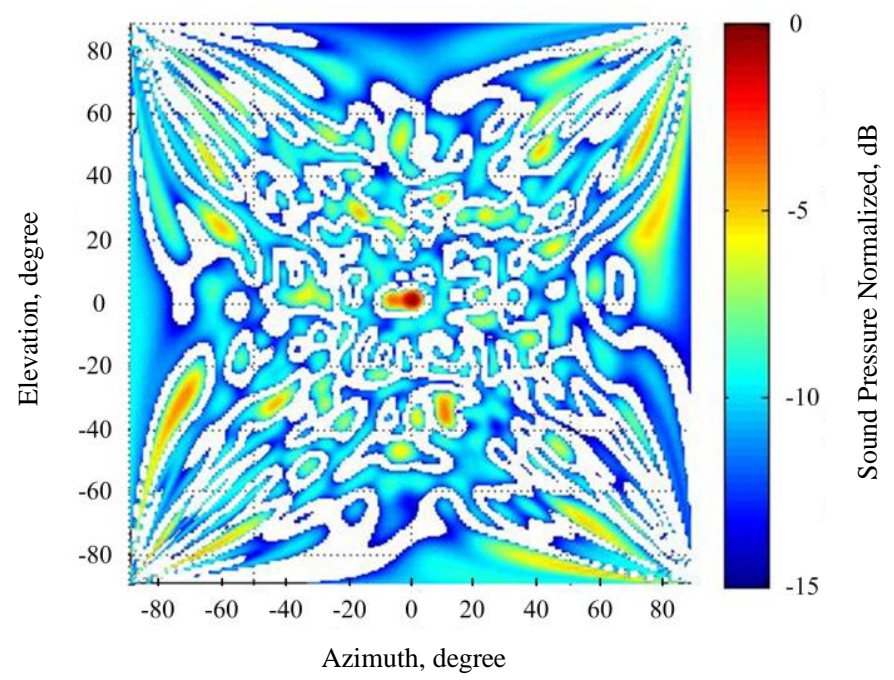

Fig. 1. Sample of generated acoustic map of two point-like sources of narrowband noise with center frequency $10 \mathrm{kHz}$

\section{Coherent mapping by bistatic radiometer}

Mapping of distant targets using their thermal electromagnetic radiation may be implemented with the help of bistatic radiometric systems. These systems are based on time difference of arrival of the radiation received by two or more antennas [3]. Coherent radiometric mapping requires synchronous scanning of antennas additionally. This scheme enables accurate range estimation and fine range resolution at the distances comparable with bistatic baseline. A mapping approach with detailed computer simulation and experimental verification is given in [3]. The minimum detectable temperature change of $0.12 \mathrm{~K}$ is obtained experimentally. The main system performance is given in Table 6 . The generated images may be tested, to be applied for estimation of vibrations.

Table 6. Performance of Ka-band bistatic radiometer

\begin{tabular}{|l|l|}
\hline Working frequencies & $36.05-36.47 \mathrm{GHz}$ \\
\hline ADC Sampling rate & $1.0 \mathrm{Gs}$ per s \\
\hline Power spectrum bandwidth at $-3 \mathrm{~dB}$ level & $0.465 \mathrm{GHz}$ \\
\hline Antenna aperture length and width & 0.7 and $0.04 \mathrm{~m}$ \\
\hline Cross-channel isolation & $\geq 70 \mathrm{~dB}$ \\
\hline Receiver gain & $97 \mathrm{~dB}$ \\
\hline Receiver noise figure & 3 \\
\hline Receiver noise temperature & $600 \mathrm{~K}$ \\
\hline
\end{tabular}

\section{Conclusions}

In this paper, a short overview of acoustic camera systems is given. The software for acoustic noise source localization and estimation which uses two methods (Delay and Sum Beamforming and Improved Beamforming in the frequency 
domain) is developed. This software generating acoustic maps was tested in indoor conditions. The performance of the purchased acoustic camera equipment was estimated at operation in the presence of multipath propagation of acoustic waves. The experimental results showed that there are specific side lobes that allow unwanted penetration. The paper gives knowledge about the position of these side lobes in order to orient the acoustic aperture correctly.

The approach for coherent radiometric mapping enables the realization of the range resolution at the distances comparable with the bistatic radiometer baseline.

The results are applicable for non-destructive testing tasks.

Acknowledgments: The research work reported in the paper was partly supported by the Project AComIn "Advanced Computing for Innovation"; Grant 316087, funded by the FP7 Capacity Programme (Research Potential of Convergence Regions) and by FP-7 Project SCOUT (Grant 607019).

\section{References}

1. Billingsley, J. An Acoustic Telescope. - Aeronautical Research Council ARC 35/364, 1974.

2. B illingsley, J., R. Kinns. The Acoustic Telescope. - Journal of Sound and Vibration, Vol. 48, 1976, pp. 485-510.

3. Lukin, K. A., V. V. Kudrias hov, P. L. Vyplavin, V. P. Pal a m a r chu k. Coherent Imaging in the Range-Azimuth Plane Using a Bistatic Radiometer Based on Antennas with Beam Synthesizing. - IEEE Aerospace and Electronic Systems Magazine, Vol. 29, 2014, Issue 7, pp. 16-22.

4. Pe i chl, M., H. S till, S. Dill, M. Gre in e r, M. J i rou se k. Imaging Technologies and Applications of Microwave Radiometry. - In: First European Radar Conference, Amsterdam, The Netherlands, 14-15 October 2004, pp. 269-273.

5. M a y n ard, J. D., E. G. W i 11 i a m s, Y. Le e. Nearfield Acoustic Holography: I. Theory of Generalized Holography and the Development of NAH. - The Journal of the Acoustical Society of America, Vol. 78, 1985, Issue 4, pp. 1395-1413.

6. Braun, K. F. Nobel Lecture: Electrical Oscillations and Wireless Telegraphy, p. 239. Nobelprize.org. Nobel Media AB 2013. Web, 28 September 2013.

7. http://www.bksv.com/

8. http://www.spectri.net/

9. IEEE Standard for a Precision Clock Synchronization Protocol for Networked Measurement and Control Systems. - IEEE Std. 1588-2002 (2002), i-144.

10. Chri st e n s e n, J. J., J. H a ld. Beamforming. - Brüel \& Kjaer Technical Review No 1, 2004, pp. 1-48.

11. V y p lavi n, P. L. Integrated and Maximal Sidelobe Levels of Noise Signal. - Applied Radio Electronics, Vol. 12, 2013, No 1, pp. 128-131.

12. Prokopenko, I. G. Statistical Synthesis of Robust Signal Detection Algorithms under Conditions of Aprioristic Uncertainty. - Cybernetics and Information Technologies, Vol. 15, 2015, No 7, pp. 13-22.

13. Ch y rk a, I. D., I. P. O me l chu k. Multichannel Modified Covariance Estimator of a SingleTone Frequency. - Cybernetics and Information Technologies, Vol. 15, 2015, No 7, pp. 35-44. 